\title{
Nonlinear front evolution of hydrodynamic chemical waves in vertical cylinders
}

\author{
Joseph W. Wilder \\ Department of Mathematics, P.O. Box 6310, West Virginia University, Morgantown, West Virginia 26506 \\ Desiderio A. Vasquez \\ Department of Physics, Indiana University-Purdue University Fort Wayne, Fort Wayne, Indiana 46805 \\ Boyd F. Edwards \\ Department of Physics, P.O. Box 6315, West Virginia University, Morgantown, West Virginia 26506
}

(Received 4 November 1996)

\begin{abstract}
The nonlinear stability of three-dimensional reaction-diffusion fronts in vertical cylinders is considered using the viscous hydrodynamic fluid equations in the limit of infinite thermal diffusivity. A nonlinear front evolution equation is presented and used to examine the transition from nonaxisymmetric to axisymmetric convection observed in experiments performed in cylinders. Comparisons with experiments show excellent agreement in both the shape and speed of the front. [S1063-651X(97)13409-2]
\end{abstract}

PACS number(s): 47.20.Bp, 03.40.Gc

\section{INTRODUCTION}

Autocatalytic reaction fronts are of interest in many fields. In systems such as iodate-arsenous acid mixtures, the exothermic front converts unreacted fluid at one density into a reacted fluid at a lower density. Experimental work on such systems $[1,2]$ has demonstrated the existence of fronts with constant curvature, moving at constant speeds. The curvature of these fronts is due to the presence of steady convective fluid motion near the reaction front. This motion is due to the hydrodynamic instability of the system, which may arise from thermal or compositional effects, acting either separately or in concert. One source of such an instability is the density difference occasioned by the chemical differences between the reacted and unreacted fluids. Among the many who have contributed to the exploration of convective fronts are Bazsa and Epstein [3] who reported in 1985 on the effect of convection on fronts, and Nagypal et al. [4] in 1986. With the arsenous-acid system, the reacted fluid has a lower density than the unreacted fluid if measured at the same reference temperature. When ascending fronts are considered, this density difference results in the lighter (reacted) fluid being below the heavier (unreacted) fluid, setting up an instability that is similar to the classical Rayleigh-Taylor instability. Hydrodynamic instability can also result from the exothermic nature of the chemical reaction, which results in the front acting as a heat source. When upward propagating fronts are considered, this heat source results in the fluid below the front being hotter than the fluid far above the front. If the temperature difference is large enough, the density gradients induced by this difference can result in a Rayleigh-Bénard-like instability. Depending on the physical system of interest, either one or both of these causes of instability can be important. As shown in a previous work [5], thermal effects are of less importance than the compositional difference between the reacted and unreacted fluids in the case of the iodate-arsenous acid system. As a result, only the relevant limiting case of infinite thermal diffusivity will be considered.
In previous works on this system $[6,7]$ the authors derived front evolution equations for the system and reported the results of simulations for a laterally unbounded system [6], as well as bounded two-dimensional geometries [7]. These front evolution equations, which are similar in nature to those used in studies of flame propagation [8], greatly facilitate the study of these complex systems by reducing the dimensionality of the partial differential equation systems that must be considered to model the phenomena. The results of these earlier studies motivated the derivation of a front evolution equation for the three-dimensional flow in a vertical cylinder to study the hypothesized transition from nonaxisymmetric to axisymmetric convection in this system. This transition was suggested by experimental observation of either axisymmetric or nonaxisymmetric fronts depending on the diameter of the capillary tube in which the experiments were performed [2]. The transition was also suggested by the results of previous studies in simpler geometries [7].

\section{EQUATIONS OF MOTION}

The full model for this system [9] involves the standard viscous hydrodynamic equations for both the reacted and unreacted fluids, with appropriate jump conditions imposed at the interface. In addition, a modified eikonal equation describes the normal velocity of the front in terms of its curvature and the fluid velocity [10]. If one applies the asymptotic analysis described in Ref. [6] to this model, one arrives at a nonlinear front evolution equation in a cylindrical geometry given by

$$
\begin{aligned}
\frac{\partial F_{0}}{\partial t}= & \mathcal{D}_{C}\left(\frac{\partial^{2} F_{0}}{\partial r^{2}}+\frac{1}{r} \frac{\partial F_{0}}{\partial r}+\frac{1}{r^{2}} \frac{\partial^{2} F_{0}}{\partial \theta^{2}}\right) \\
& +\frac{v_{0}}{2}\left[\left(\frac{\partial F_{0}}{\partial r}\right)^{2}+\frac{1}{r^{2}}\left(\frac{\partial F_{0}}{\partial \theta}\right)^{2}\right]+w_{0}(r, \theta, 0, t),
\end{aligned}
$$

with

3016

(C) 1997 The American Physical Society 


$$
\frac{\partial F_{0}}{\partial r}=0, \quad r=1
$$

where $F_{0}$ represents the deviation of the front from a planar geometry in a nondimensional form where we have used length and time measured in units of $a$ and $a^{2} / \nu$, where $a$ represents the tube radius.

Since a planar front would propagate with a speed $c_{0}$, which is easily obtained from experiments, the asymptotics yield a dimensioned speed for the nonplanar front given by $c_{0}+(\nu / a) \partial F_{0} / \partial t$. In Eq. (1a), $\mathcal{D}_{C}=D_{C} / \nu$ and $v_{0}=a c_{0} / \nu$, where $D_{C}$ is the chemical diffusivity and $\nu$ is the fluid viscosity. The last term on the right-hand side is the vertical component of the fluid velocity evaluated at the front, and is obtained from solving the following system:

$$
\begin{gathered}
-v_{0} \frac{\partial \vec{v}}{\partial z}=-\vec{\nabla} p_{0}+\nabla^{2} \vec{v}, \\
\vec{\nabla} \cdot \vec{v}=0,
\end{gathered}
$$

with jump conditions

$$
\begin{gathered}
{\left[w_{0}\right]_{-}^{+}=0,} \\
{\left[\frac{\partial w_{0}}{\partial z}\right]_{-}^{+}=0,} \\
{\left[\frac{\partial^{2} w_{0}}{\partial z^{2}}\right]_{-}^{+}=0,} \\
{\left[p_{0}\right]_{-}^{+}=-\delta G F_{0},}
\end{gathered}
$$

where $\vec{v}(x, z)=u_{0}(r, \theta, z) \hat{\vec{r}}+v_{0}(r, \theta, z) \hat{\theta}+w_{0}(r, \theta, z) \hat{\vec{z}}, \quad G$ $=g a^{3} / \nu^{2}$, with $g$ being the acceleration of gravity and the notation $[q]_{-}^{+}$denotes the jump in $q$ across the interface between the reacted and unreacted fluids. The parameter $\delta$ in Eq. (2f) is the relative density difference between the unreacted and the reacted fluids.

Equation (1a) represents a nonlinear equation for the propagation of the front, which is coupled to the fluid flow only through the last term on the right-hand side, which is the vertical velocity of the fluid at the front. Equations (2a)(2f) can be solved to find $w_{0}(r, \theta, 0, t)$, to be used in Eq. (1a).

The problem of determining $w_{0}(r, \theta, 0, t)$ can be simplified by doing a mode expansion of the fluid velocities and related quantities. In doing so, there is an axisymmetric and nonaxisymmetric portion of the variables. The problem associated with the axisymmetric portion of the fluid velocity reduces to that of solving the linear stability problem of axisymmetric flow in a cylinder with the exception that the jump condition on the pressure is determined by the solution of Eq. (1a) at the previous time step instead of being given by the solution of a linearized version of (1a) as would be done in the linear stability problem. This was considered in a previous work [7], with the details of the method given in Ref. [11].

The nonaxisymmetric portion of the problem similarly reduces to that of the linear stability of nonaxisymmetric flow in a cylinder with the same difference in the specification of the jump condition on the pressure as discussed above. After performing the mode expansion, the appropriate eigenfunctions can be obtained as discussed in Ref. [12].

Since we are interested in studying the transition from nonaxisymmetric to axisymmetric convection, and since a previous work [12] found that the onset of convection involved mode $m=1$ (i.e., functional dependence of the solutions on $\cos \theta$ and $\sin \theta$ ), we assume

$$
\begin{gathered}
F_{0}(r, \theta, t)=f^{(0)}(r, t)+f_{c}^{(1)}(r, t) \cos \theta+f_{s}^{(1)}(r, t) \sin \theta, \\
w_{0}(r, \theta, z, t)=w^{(0)}(r, z, t)+w_{c}^{(1)}(r, z, t) \cos \theta \\
+w_{s}^{(1)}(r, z, t) \sin \theta,
\end{gathered}
$$

and analogous forms for the other dependent variables. Substitution into Eqs. (1) and separation of the different modes lead to

$$
\begin{aligned}
\frac{\partial f^{(0)}}{\partial t}= & \mathcal{D}_{C}\left(\frac{\partial^{2} f^{(0)}}{\partial r^{2}}+\frac{1}{r} \frac{\partial f^{(0)}}{\partial r}\right)+\frac{v_{0}}{2}\left[\left(\frac{\partial f^{(0)}}{\partial r}\right)^{2}+\frac{1}{2}\left(\frac{\partial f_{c}^{(1)}}{\partial r}\right)^{2}\right. \\
+ & \left.\frac{1}{2 r^{2}}\left(f_{c}^{(1)}\right)^{2}\right]+w^{(0)}(r, 0, t), \\
\frac{\partial f_{c}^{(1)}}{\partial t}= & \mathcal{D}_{C}\left(\frac{\partial^{2} f_{c}^{(1)}}{\partial r^{2}}+\frac{1}{r} \frac{\partial f_{c}^{(1)}}{\partial r}-\frac{1}{r^{2}} f_{c}^{(1)}\right)+v_{0} \frac{\partial f^{(0)}}{\partial r} \frac{\partial f_{c}^{(1)}}{\partial r} \\
& +w_{c}^{(1)}(r, 0, t), \\
\frac{\partial f_{s}^{(1)}}{\partial t}= & \mathcal{D}_{C}\left(\frac{\partial^{2} f_{s}^{(1)}}{\partial r^{2}}+\frac{1}{r} \frac{\partial f_{s}^{(1)}}{\partial r}-\frac{1}{r^{2}} f_{s}^{(1)}\right)+v_{0} \frac{\partial f^{(0)}}{\partial r} \frac{\partial f_{s}^{(1)}}{\partial r} \\
& +w_{s}^{(1)}(r, 0, t),
\end{aligned}
$$

with

$$
\begin{gathered}
\frac{\partial f^{(0)}}{\partial r}=0, \quad r=0,1, \\
\frac{\partial f_{c}^{(1)}}{\partial r}=\frac{\partial f_{s}^{(1)}}{\partial r}=0, \quad r=1
\end{gathered}
$$

and where the necessary fluid velocities are determined as described above (see Refs. [11] and [12] for details). The solution procedure is then as follows: given some initial conditions for $f^{(0)}, f_{c}^{(1)}$, and $f_{s}^{(1)}$, we compute $w^{(0)}, w_{c}^{(1)}$, and $w_{s}^{(1)}$. These are then used in Eqs. (4), and the front position at the next time step computed using a standard explicit finite differencing of Eqs. (4), as has been used previously for similar front evolution equations [6,7].

\section{RESULTS AND DISCUSSION}

In the results reported below, we have used $D_{C}=2.0$ $\times 10^{-5} \mathrm{~cm}^{2} / \mathrm{s}, \quad \nu=9.2 \times 10^{-3} \mathrm{~cm}^{2} / \mathrm{s}, \quad \delta=0.87 \times 10^{-4}$, and except where noted, $c_{0}=2.95 \times 10^{-3} \mathrm{~cm} / \mathrm{s}$. The time step and cell discretization size arising from the finite differencing of Eqs. (4) were adjusted to ensure the accuracy and convergence of the solutions. It was observed that after the decay of transients due to the initial conditions, the front 


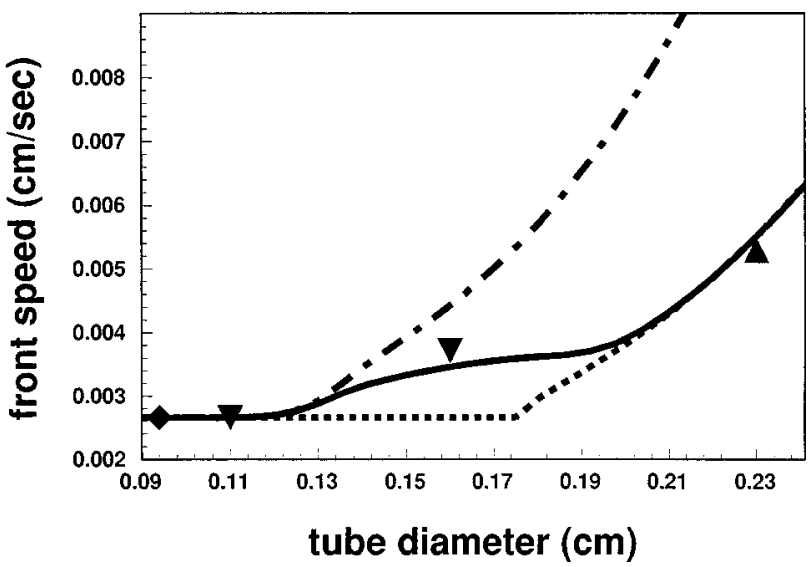

FIG. 1. Comparison of theoretical calculations for a cylinder with experimental measurements of the front speed as a function of tube diameter in vertical capillary tubes. Solid diamonds represent experimentally observed flat fronts; solid downward-pointing triangles represent nonaxisymmetric observed fronts, and solid upward-pointing triangles represent axisymmetric observed fronts. Shown are results of computations only allowing axisymmetric fluid flow (dotted trace), only allowing nonaxisymmetric flow (chain-dashed trace), and the full calculation where the system is allowed to evolve without any such constraints on the nature of the fluid flow (solid trace).

assumed a constant shape and speed. It is interesting to note that the linear stability theory predicted that the onset of convection in a cylinder should be nonaxisymmetric [11], while the original experimental work on this system showed only axisymmetric fronts in cylinders [1]. The nonaxisymmetric nature of the front was also predicted by Pojman and Epstein [13], though for a simplified system. Recent experimental results [2] have shown that near the onset of convection the fronts are nonaxisymmetric, with axisymmetric fronts observed in cylinders with a diameter of $0.23 \mathrm{~cm}$ and larger.

Figure 1 shows the computed steady speed of the constant-shape fronts, which evolve from the above model after transients due to the random initial conditions have decayed. Note that we have converted back to dimensioned units after reconstructing the front using Eqs. (3) to facilitate comparison with experiments. Shown in the figure are the results of only allowing axisymmetric fluid flow (dotted trace), only allowing nonaxisymmetric flow (dot-dashed trace), and the full calculation where the system is allowed to evolve without any such constraints (solid trace). Also shown are the experimental results from Ref. [2] when the front was flat (diamond), nonaxisymmetric (downward pointing triangles), and axisymmetric (upward pointing triangle). We note in this figure the strikingly good agreement between the experimental and computed front velocities. We also note the influence of axisymmetric convection on the speed of the front prior to reaching the tube diameter corresponding to the onset of axisymmetric convection in the absence of the nonaxisymmetric modes. This is obviously the result of the nonlinearities in the problem.

Figure 2 shows a comparison of an experimentally observed front [Fig. 2(a)] reported in Ref. [2] and one obtained computationally [Fig. 2(b)] when the tube diameter is 0.16 $\mathrm{cm}$. Also shown in this figure is a front obtained computa-
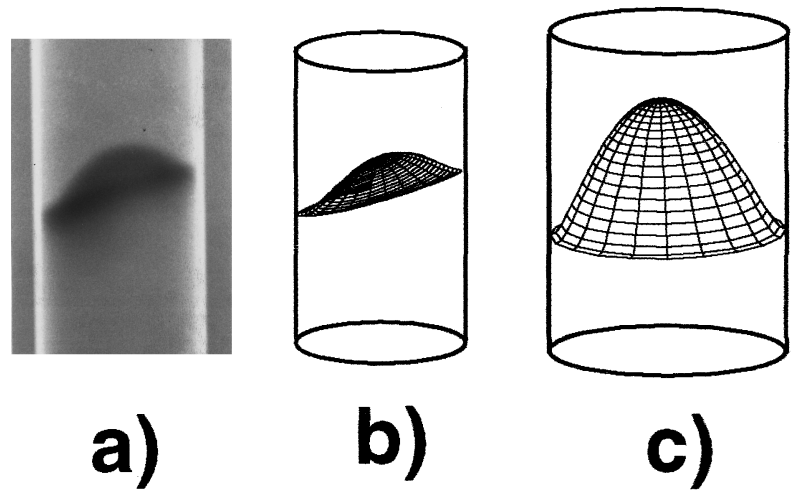

FIG. 2. Shown are an experimental front in a tube with diameter $0.16 \mathrm{~cm}(\mathrm{a})$, a computed front in a tube of diameter $0.16 \mathrm{~cm} \mathrm{(b),} \mathrm{and}$ a computed front in a tube of diameter $0.23 \mathrm{~cm}(\mathrm{c})$.

tionally when the tube diameter is $0.23 \mathrm{~cm}$ [Fig. 2(c)], which shows the axisymmetric shape reported in the literature for this diameter.

Such close agreement between the speed and shape of the fronts as evidenced by Figs. 1 and 2 is especially surprising since the parameter values used are in some cases only estimates (e.g., $D_{C}=2.0 \times 10^{-5} \mathrm{~cm}^{2} / \mathrm{s}, \nu$ being that of pure water, etc.); no fitting of the model parameters to the experimental data was necessary. This simplified model seems to have accurately captured the transition from nonaxisymmetric to axisymmetric convection. As can be seen from Fig. 1, there is a crossover region that represents a mixed-mode solution. The shape of the fronts observed experimentally in this region [Fig. 2(a), for example] supports this since it is obviously neither purely axisymmetric nor nonaxisymmetric, but a mixture of the two.

As a further test of the theory, we can use the data available from a recent work [2] on the speed of nonaxisymmetric fronts in a cylinder (of diameter $0.14 \mathrm{~cm}$ ) as a function of iodate concentration. In our model, we can write $\delta=1.74$ $\times 10^{-2} M^{-1}\left[\mathrm{IO}_{3}{ }^{-}\right] .^{2}$ From reaction-diffusion theory, the speed of the convectionless front is given by $c_{0}=a_{T}$ $+b_{T}\left[\mathrm{IO}_{3}{ }^{-}\right]$, where $a_{T}=7.45 \times 10^{-6} \mathrm{~cm} \mathrm{~s}^{-1}$ and $b_{T}$ $=0.373 \mathrm{~cm} \mathrm{~s}^{-1} M^{-1}[2]$. A recent work found experimental

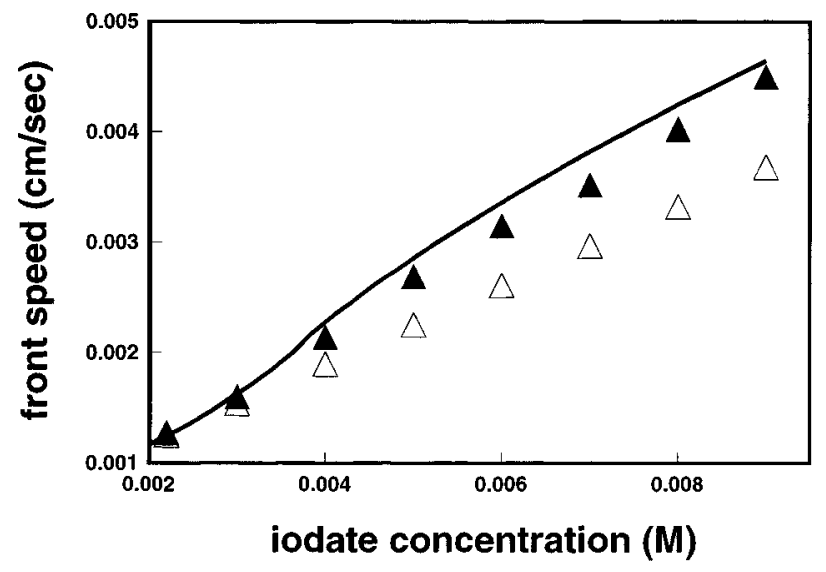

FIG. 3. Shown is the front speed as a function of iodate concentration. Represented by solid (open) triangles are experimental results for upward (downward) propagation in tubes, as well as the theoretical predictions for upward propagation, solid line. 
values of $a_{E}=4.69 \times 10^{-4} \mathrm{~cm} \mathrm{~s}^{-1}$ and $b_{E}=0.357$ $\mathrm{cm} \mathrm{s}^{-1} M^{-1}$ [2]. These results are shown in Fig. 3 where the open triangles represent the experimental speeds for the convectionless fronts (downward propagation of the front). Also shown are the experimentally observed front speeds when convection was present (upward propagation, solid triangles) and the results of our numerical calculations (solid trace) using the values of $c_{0}$ found experimentally. The agreement evidenced in this figure between experimental and computational front speeds helps to further validate the model. We note that while not discussed in the experimental literature, a transition is also observed from nonaxisymmetric to axisymmetric convection as the iodate concentration is varied. This is not surprising since the iodate concentration affects the parameter $\delta$, which in turn affects the stability properties of the underlying fluid flow. By changing the iodate concentration we are thus changing the length scale associated with the problem, and thereby allow the observation of a transition from nonaxisymmetric to axisymmetric convection, even though the diameter of the tube is fixed.

\section{CONCLUSIONS}

A front evolution equation for chemical waves in a cylindrical geometry has been presented. In a region near the reacting front, asymptotics reduce the hydrodynamic equations to a linear system, which is solved for the fluid velocity at the front in terms of the front position. When this is used in the nonlinear equation describing the front's evolution, the result is a single equation involving the front position, which also contains the essential hydrodynamics of the system. Calculations predict experimental observations in tubes near the onset of convection, as well as sufficiently far away from the onset such that the observed convection is axisymmetric. This simplified model accurately predicts the shape and speed of the front, without having to solve the very complicated three-dimensional nonlinear Navier-Stokes equations. It also captures the transition from nonaxisymmetric to axisymmetric convection observed in experiments. This method should also prove useful in other reaction-diffusion systems involving front propagation.

\section{ACKNOWLEDGMENTS}

J.W.W. and B.F.E. would like to acknowledge the support of this work by NSF Grant No. OSR-9255224. The authors would also like to thank Kenneth Showalter for providing the experimental data used for comparison with the calculations presented here.
[1] T. McManus, Ph.D. thesis, West Virginia University (unpublished), Chap. 3, 1989.

[2] J. Masere, D. A. Vasquez, B. F. Edwards, J. W. Wilder, and K. Showalter, J. Phys. Chem. 98, 6505 (1994).

[3] G. Bazsa and I. R. Epstein, J. Phys. Chem. 89, 3050 (1985).

[4] I. Nagypal, G. Bazsa, and I. R. Epstein, J. Am. Chem. Soc. 108, 3635 (1986).

[5] J. W. Wilder, B. F. Edwards, and D. A. Vasquez, Phys. Rev. A 45, 2320 (1992).

[6] J. W. Wilder, B. F. Edwards, D. A. Vasquez, and G. I. Sivashinsky, Physica D 73, 217 (1994).

[7] J. W. Wilder and D. A. Vasquez, Physica D 90, 170 (1996).
[8] G. I. Sivashinsky, Acta Astron. 4, 1177 (1977).

[9] B. F. Edwards, J. W. Wilder, and K. Showalter, Phys. Rev. A 43, 749 (1991).

[10] J. W. Wilder, D. A. Vasquez, and B. F. Edwards, Phys. Rev. E 47, 3761 (1993).

[11] D. A. Vasquez, B. F. Edwards, and J. W. Wilder, Phys. Rev. A 43, 6694 (1991).

[12] D. A. Vasquez, J. W. Wilder, and B. F. Edwards, Phys. Fluids A 4, 2410 (1992).

[13] J. A. Pojman and I. R. Epstein, J. Phys. Chem. 94, 4966 (1990). 